\title{
Reconocimiento preliminar de la densidad y estructura poblacional de Saguinus tripartitus Milne-Eduards en la Amazonía peruana
}

\section{Preliminary survey on population density and structure of Saguinus tripartitus Milne-Eduards from the Peruvian Amazon}

\author{
Rolando Aquino ${ }^{1}$, Carlos Ique ${ }^{2}$ y Hugo Gálvez ${ }^{2}$
}

Presentado: 20/10/2005

Aceptado: $\quad 12 / 01 / 2005$

\section{Resumen}

Censos por transecto fueron llevados a cabo en enero del 2005 en la cuenca del río Aushiri para determinar la densidad poblacional y estructura poblacional del «pichico dorado» (Saguinus tripartitus MilneEduards). Fueron registrados 17 grupos, cuya densidad poblacional fue calculada en 13,5 individuos $/ \mathrm{km}^{2}$. El tamaño de los grupos fluctuaron desde cuatro a ocho individuos y el tamaño promedio fue 5,3 individuos/grupo. Cada grupo estuvo integrado por la pareja reproductora y su progenie de dos a seis individuos. Los adultos conformaron el $75 \%$ de la población.

Palabras claves: Pichico dorado, censos, densidad poblacional, tamaño de grupo, estructura poblacional.

\section{Abstract}

Censuses by transects were carried out in January of the 2005 in the Aushiri river basin to determine the population density and population structure of the «golden-mantle tamarin» (Saguinus tripartitus MilneEduards). We recorded 17 groups and population density was calculated in 13,5 individuals $/ \mathrm{km}^{2}$. The size of the groups varied from four to eight individuals, with an average size of 5,3 individuals per group. The basic composition of a group was that of a reproductive pair with progeny of two to six individuals. The adults represented $75 \%$ of the total population.

Keywords: Golden tamarin, census, population density, group size, population structure.

\section{Introducción}

De acuerdo con la última lista de especies de primates reconocidas para el nuevo mundo (Rylands et al., 2000), en la Amazonía peruana se encuentran habitando siete especies del género Saguinus. De ellas, tres tienen por distribución el nororiente peruano, entre ellas Saguinus tripartitus, considerada hasta 1987 como una subespecie de S. fuscicollis Spix (Hershkovitz, 1977) y posteriormente reconocida por Thorington (1988) como una especie distinta. Entre los callitrícidos, S. tripartitus, con distribución restringida en Ecuador a los ríos Napo y Curaray (Albuja, 1994) y Perú al

\footnotetext{
${ }^{1}$ Instituto de Ciencias Biológicas Antonio Raimondi (ICBAR)/ Facultad de Ciencias Biológicas, Universidad Nacional Mayor de San Marcos. Rolando Aquino, P.O. Box 575, Iquitos, Perú. E-mail Rolando Aquino: raquinoy2005@yahoo.es

${ }^{2}$ Instituto Veterinario de Investigaciones Tropicales y de Altura (IVITA)/ Facultad de Medicina Veterinaria, Universidad Nacional Mayor de San Marcos.
}

curso superior de ambas márgenes del río Napo hasta las cabeceras del río Santa María en la margen izquierda y río Curaray en la margen derecha (Aquino y Encarnación, 1996), es una de las pocas especies que aún no han sido estudiadas en detalle, en particular en lo que se refiere a la ecología, comportamiento y dinámica poblacional, entre otros. Es oportuno resaltar que entre los ríos Napo y Curaray, $S$. tripartitus es el único representante del género Saguinus, mientras que en la margen izquierda del curso superior del río Napo, estaría compartiendo el área con Saguinus graellsi Jiménez de la Espada.

Las escasas referencias basadas en observaciones de corta duración dan cuenta que esta especie vive en grupos de hasta 10 individuos (Albuja, 1994) y realizan sus actividades mayormente en el estrato más bajo del bosque (Heymann, 2000). La dieta alimenticia consis- 
te de frutos, insectos y néctar (Aquino y Encarnación, 1994, Kostrub, 1997), pero también de flores y exudados de goma (Heymann, 2000) e incluso del raquis seco y foliolos de helechos, así como del corcho de las Sapotáceas y Bombacáceas (Aquino y Encarnación, 1994).

La falta de información en lo que concierne a densidad y estructura poblacional para esta especie, nos motivó a la conducción de censos por transectos y observaciones adicionales llevados a cabo entre el 05 al 29 de enero del 2005 en los ríos San Joséy Aushiri, localizados entre las cuencas de los ríos Napo y Curaray. Los resultados obtenidos se presentan en este documento.

\section{Métodos}

\section{Área de estudio}

El área de estudio se encuentra localizada entre los ríos Napo y Curaray, en el límite fronterizo con el Ecuador y comprendió los bosques aledaños a los ríos San José y Aushiri (Fig. 1). El área correspondió al típico bosque de altura, donde se diferenció algunas formaciones vegetales como bosque de terraza alta, bosque de tahuampa de altura, bosque de colina baja, bosque de galería y aguajal de altura.

En los bosques de terraza alta y colina baja, la vegetación estuvo representada por árboles de porte alto, entre 25 a $30 \mathrm{~m}$, con algunos emergentes de hasta $40 \mathrm{~m}$, destacándose por su mayor presencia Oenocarpus bataua Burret, Micropholis sp., Pouteria sp., Couma macrocarpa Barb, Rodr., Hymenaea sp., Parkia sp., entre otras. El Sotobosque se caracterizó por ser moderadamente abierto y estuvo poblado entre otras por plantas de Lepidocaryum sp. y Attalea sp.

En los bosques de galería y tahuampa de altura, la vegetación estuvo conformada por árboles de porte mediano entre 20 a $25 \mathrm{~m}$ de alto, con algunos emergentes hasta $30 \mathrm{~m}$, muchos de ellos cubierto por un enmarañado de bejucos y lianas. Entre las plantas de mayor frecuencia se observaron Bactris sp., Socratea sp., Pourouma sp., Rheedia sp., Astrocaryum sp., Inga spp., Cecropia spp., Ficus antihelmintica Mart, entre otras. A diferencia del anterior, el sotobosque se caracterizó por ser bastante denso por la presencia de enmarañados de bejucos y lianas y por plantas her-

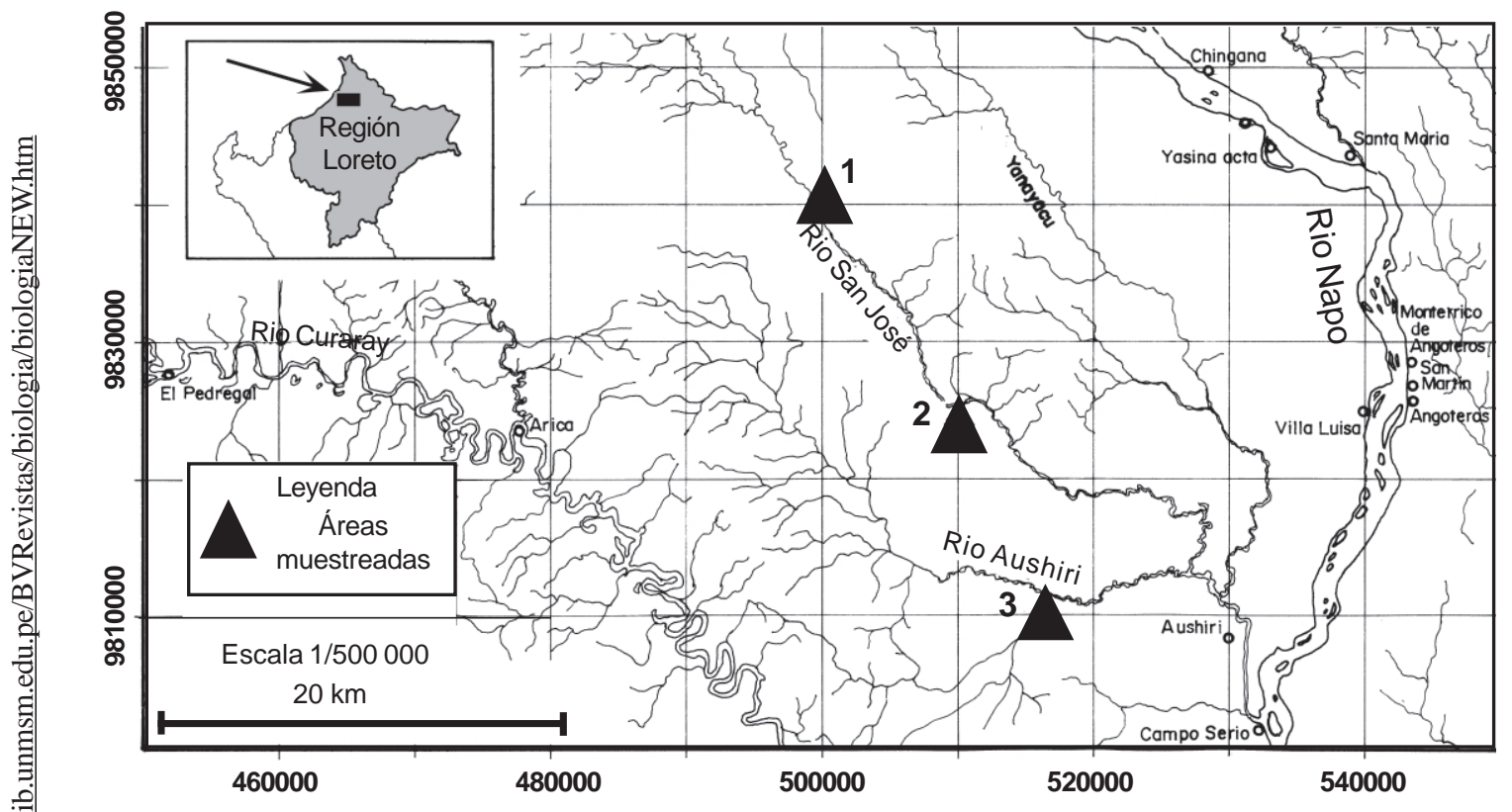

Figura 1. Ubicación geográfica del área de estudio de Saguinus tripartitus en el Noreste de la Amazonía peruana. 
báceas perennes como Heliconia sp. y representantes de las Musáceas y Zingiberáceas.

Los aguajales de altura fueron comunes en el área de estudio y se caracterizaron por su disposición en fajas de hasta $1 \mathrm{~km}$ de largo y entre 50 a $200 \mathrm{~m}$ de ancho. Estos aguajales estuvieron poblados principalmente por Mauritia flexuosa L. f, Euterpe oleraceae Mart, Iriartea exorrhiza Mart.y Ficus spp.

En términos generales, el área de estudio presentó un aspecto bastante alterado por la presencia de numerosas trochas y campamentos en uso y el constante trajinar de cazadores y extractores de productos forestales. De lo anterior, se deduce que la fauna silvestre estuvo sujeta a una persistente caza; no obstante y a diferencia de lo que ocurre en otras cuencas de la Amazonía peruana, todavía se encuentran ungulados y primates de tamaño grande y mediano, por lo que la caza aún no incluye a primates de tamaño pequeño.

\section{Censos}

Para los censos fueron definidos tres estaciones de muestreo, dos en el río San José y uno en el río Aushiri. Considerando el corto tiempo disponible, hicimos uso de las trochas existentes con fines de caza, cuyas longitudes variaron entre 3,0 a 4,0 km de longitud. En cada estación de muestreo fueron recorridas de dos a tres trochas, cada una en dos oportunidades. Los censos fueron conducidos por dos grupos, cada uno de dos observadores, quienes se desplazaron a una velocidad de $1,5 \mathrm{~km} /$ h, desde las 0700 a 1100 y de 1400 a 1700 h (hora local). Cada vez que hubo avistamiento procedimos a anotar en la libreta de campo la especie, tamaño de grupo, altura en el árbol del primer individuo avistado, tipo de vegetación circundante y la distancia perpendicular del primer individuo avistado al transecto. Cuando se trataba de $S$. tripartitus, se determinó la composición del grupo, mediante el seguimiento y observación del grupo; aún así, fue muy difícil diferenciar los machos de las hembras por la conducta huidiza y arisca en la mayoría de los casos. No obstante, fue posible
Tabla 1. Densidad poblacional de Saguinus tripartitus para la cuenca del río Aushiri, afluente derecho del río Napo.

\begin{tabular}{lcc}
\hline Densidad & \%CV & $\begin{array}{r}\text { 95\% intérvalo } \\
\text { de confianza }\end{array}$ \\
\hline 2,4 grupos $/ \mathrm{km}^{2}$ & 24,0 & 1,7 \\
13,5 individuos $/ \mathrm{km}^{2}$ & 25,0 & 7,7 \\
\hline
\end{tabular}

diferenciar a los juveniles, que se caracterizaron por su locomoción propia y por tener aún los bigotes blanquecinos y a los infantes I que carecían de locomoción propia. La densidad poblacional fue estimada usando el Programa Distance, versión 4,0, método ampliamente utilizado en la evaluación de poblaciones de fauna silvestre tropical (Buckland et al. 1993, Laake et al. 1994, Wilson et al. 1996).

\section{Resultados}

En $132 \mathrm{~km}$ de longitud censada fueron observados 17 grupos de $S$. tripartitus. De ellos, 11 ocurrieron en bosques de terraza alta y 6 en bosques de galería. La densidad poblacional para el área de estudio fue estimada en 2,4 grupos/ $\mathrm{km}^{2}$ y 13,5 individuos $/ \mathrm{km}^{2}$ (Tabla 1 ).

En 14 de los 17 grupos observados se logró el conteo completo. El tamaño de los grupos completos varió de cuatro a ocho individuos, con un promedio de 5,3 individuos por grupo (Tabla 2). Los grupos de mayor frecuencia de observación fueron de cuatro individuos (seis grupos ó $43 \%$ del total) y seis individuos (cuatro grupos ó 29\% del total).

Pese a las dificultades antes mencionadas,

Tabla 2. Grupos de Saguinus tripartitus observados en la cuenca del río Aushiri, afluente derecho del río Napo.

\begin{tabular}{lccc}
\hline $\begin{array}{l}\text { Tamaño } \\
\text { de grupo }\end{array}$ & $\begin{array}{c}\text { Frecuencia } \\
\text { observada }\end{array}$ & $\begin{array}{c}\text { Total } \\
\text { individuos }\end{array}$ & $\%$ \\
\hline 4 & 6 & 24 & 43 \\
5 & 1 & 5 & 7 \\
6 & 4 & 24 & 29 \\
7 & 2 & 14 & 14 \\
8 & 1 & 8 & 7 \\
Total: & 14 & 75 & 100 \\
\hline
\end{tabular}


Tabla 3. Composición de 14 grupos completos de Saguinus tripartitus observados en la cuenca del río Aushiri, afluente derecho del río Napo.

\begin{tabular}{|c|c|c|c|c|}
\hline Grupos & Adultos & Juveniles & Infante I & Total \\
\hline$\overline{1}$ & 3 & 1 & - & 4 \\
\hline 2 & 4 & 1 & 1 & 6 \\
\hline 3 & 4 & 1 & 1 & 6 \\
\hline 4 & 3 & 1 & - & 4 \\
\hline 5 & 4 & 1 & 2 & 7 \\
\hline 6 & 3 & 1 & - & 4 \\
\hline 7 & 6 & 1 & - & 7 \\
\hline 8 & 3 & 1 & - & 4 \\
\hline 9 & 4 & 1 & 1 & 6 \\
\hline 10 & 4 & - & - & 4 \\
\hline 11 & 4 & 1 & - & 5 \\
\hline 12 & 7 & 1 & - & 8 \\
\hline 13 & 3 & 1 & - & 4 \\
\hline 14 & 5 & - & 1 & 6 \\
\hline
\end{tabular}

se puede sostener que la composición básica del grupo estuvo conformada por la pareja reproductiva y su progenie integrada desde uno a cuatro adultos, un juvenil y desde uno a dos infantes I (Tabla 3).

La estructura poblacional fue analizada en base a 75 individuos pertenecientes a 14 grupos completos. De las tres categorías establecidas para este propósito, los adultos representaron el $75 \%$ de la población, mientras que los infantes I apenas alcanzaron el 9\% de la población total. En resumen, la proporción entre adultos e inmaduros en la población total observada fue de 75:25 (Tabla 4)

\section{Discusión}

\section{Densidad poblacional}

La falta de información sobre densidades en otras áreas de la distribución geográfica de Saguinus tripartitus no permite realizar com-

Tabla 4. Estructura poblacional de 14 grupos completos de Saguinus tripartitus observados en la cuenca del río Aushiri, afluente derecho del río Napo.

\begin{tabular}{lcc}
\hline Edades & No. Individuos & $\%$ \\
\hline Adultos & 56 & 75,0 \\
Juveniles & 12 & 16,0 \\
Infantes I & 7 & 9,0 \\
Total: & 75 & 100,0 \\
\hline
\end{tabular}

paraciones, salvo los resultados con otras especies del mismo género. Nuestro resultado fue mayor al reportado para S. fuscicollis de la cuenca del río Pucacuro, afluente izquierdo del río Tigre, cuyo estimado fue de 3,0 individuos/ $\mathrm{km}^{2}$ (Aquino et al., 2000), pero menor a los reportados para S. fuscicollis y Saguinus mystax Spix en la cuenca del río Manití, donde las densidades estimadas fueron de 28 individuos $/ \mathrm{km}^{2}$ y de 25 individuos $/ \mathrm{km}^{2}$, respectivamente (Soini \& Cóppula, 1995) y para Saguinus labiatus E. Geoffroy, habitante del suroriente peruano, cuya densidad fue estimada en 18,6 individuos $/ \mathrm{km}^{2}$ (Puertas et al. (1995). Considerando la similitud de hábitat entre una y otra área, la baja densidad estimada para S. fuscicollis en el Pucacuro, podría estar asociada a la presencia de otros primates competidores por los mismos recursos alimenticios. Caso similar ocurrió en la cuenca del río Samiria, donde Aquino et al. (2001) estimó mayor densidad para $S$. fuscicollis en zonas de persistente caza y menor densidad en zonas de moderada y ligera caza. La mayor densidad estimada para S. fuscicollis y S. mystax en el Manití estaría relacionado con la escasa presencia de primates de tamaño mediano y grande, debido a la alta presión de caza y mayor alteración del hábitat por la practica de tala del bosque con fines agrícolas, actividad de la que han sacado mayor provecho los callitrícidos, quienes demostraron mejor adaptación a las condiciones que ofrecen los bosques secundarios, caracterizados por parcelas abandonadas, donde la oferta de alimento incluyen los productos de plantas frutales de diversas especies. Finalmente, la mayor densidad estimada para S. labiatus en el suroriente peruano tendría relación con la diferencia en la metodología empleada para la estimación de la densidad de esa especie.

\section{Tamaño de grupo}

El rango de variación de tamaño de grupo para $S$. tripartitus fue similar al reportado por Albuja (1994), Kostrub (1997), Heymann (2000) y Heyman et al. (2002), aún cuando en 
esta oportunidad no se registró grupos de 9 y 10 individuos. Al respecto, Kostrub (1997), menciona que el grupo de 10 individuos en realidad se trataba de una asociación temporal de dos grupos compuestos cada uno de cinco individuos. Esta conducta poco usual también fue observado años atrás en la especie $S$. mystax, donde un aparente grupo de 12 individuos capturado, correspondían en realidad a dos grupos de siete y cinco individuos, respectivamente. Otros reportes de asociaciones temporales intraespecíficas han sido reportados para S. nigricollis Spix (Izawa, 1978) y $S$. fuscicollis (Castro y Soini, 1977). La confirmación o no de grupos de hasta 10 individuos ó más en $S$. tripartitus solo podrá ser despejada con estudios más detallados. De lo que no hay duda es que en los callitrícidos, los grupos mayormente fluctúan entre cuatro a siete individuos y raramente arriba de ocho individuos. El tamaño promedio de grupo estimado para este estudio fue menor al reportado por Albuja (1994) y Kostrub (1997) cuyos promedios fueron estimados en base a seis y nueve grupos, respectivamente. El tamaño promedio estimado también fue muy cercano al reportado por Glander et al. (1984) y Soini \& Cóppula (1995) para $S$. mystax y $S$. fusciicollis, pero menor al reportado por Freese et al. (1982) para $S$. mystax y Pook \& Pook (1982), Castro et al. (1990) y Puertas et al. (1995) para S. labiatus y S. fuscicollis. La fluctuación del tamaño promedio de grupo podría estar influenciada con la temporada de pariciones, aún cuando Soini \& Cóppula (1995) sostienen que la variación del tamaño de grupo mayormente ocurre unos meses antes del inicio de pariciones con el retiro de algún miembro del grupo.

\section{Organización social y Estructura poblacional}

Los adultos se diferenciaron de los demás por el tamaño y porque casi siempre se movilizaban a la vanguardia del grupo. Los juveniles de tamaño mucho menor que el de un adulto, pero sin los bigotes largos que caracterizan a los infantes, generalmente se movilizaban detrás de los adultos. En ninguno de los grupos se observaron infantes II, ausencia que estaría asociada a la época en que se ejecutaron los censos, los que coincidieron con la temporada de pariciones, reportado para esta parte de la Amazonía peruana entre los meses de noviembre a enero (Aquino y Encarnación, 1990). Finalmente, en los grupos familiares con presencia de infantes I, éstos eran transportados por un adulto. La presencia de un solo infante en la mayoría de grupos con crías, hace suponer que la pérdida de los vástagos por una u otra razón ocurre a temprana edad, acontecimiento que parece común incluso en otras especies del mismo genero como $S$. fuscicollis y $S$. mystax. Resultados similares fueron obtenidos por Soini \& Cóppula (1995) para S. fuscicollis y $S$. mystax y por Puertas et al. (1995) para $S$. labiatus, lo que indicaría que en los callitrícidos, esta edad probablemente es la más crítica para la supervivencia del individuo, quizá porque en el transporte y cuidado, además de los progenitores también participan el resto de los componentes del grupo (Goldizen, 1987; Heymann, 1990), por lo tanto no habría una protección adecuada.

\section{Agradecimientos}

Este estudio fue realizado gracias al apoyo económico del Proyecto Peruano de Primatología «Manuel Moro Sommo»con sede en Iquitos y bajo la dirección del Dr. Enrique Montoya G. Nuestra gratitud a los jefes de las comunidades de Camposerio y Aushiri, por habernos permitido el ingreso a los ríos Aushiri y San José, jurisdicciones de las comunidades nativas de los quichuas. Especial deferencia a los hermanos César Coquinche y Arbildo Coquinche, quienes nos brindaron su apoyo incondicional como guías de campo durante los censos.

\section{Literatura citada}

Albuja, L. 1994. Nuevos registros de Saguinus tripartitus en la Amazonía Ecuatoriana. Neotropical Primates 2(2): $8-10$.

Aquino, R. y F. Encarnación. 1994. Primates of Peru/ Los Primates del Perú. Primate Report 40: 1 - 127.

Aquino, R. y F. Encarnación. 1996. Distribución geográ- 
Aquino et al.

fica de Saguinus tripartitus en la Amazonía del Perú. Neotropical primates 4(1): 1 - 4.

Aquino, R.; R. E. Bodmer y E. Pezo. 2000. Evaluación de primates en la cuenca del río Pucacuro, Amazonía peruana. En: F. San Martín y M. García (ed): La Primatología en el Perú, Vol. II, Lima, Perú, 92 - 100.

Aquino, R.; R. E. Bodmer y J. G. Gil. 2001. Mamíferos de la Cuenca del Río Samiria: Ecología Poblacional y Sustentabilidad de la Caza. Imprenta Rosegraff, Lima, Perú.

Buckland, S.; D. Anderson; K. Burnham y J. Laake. 1993. Distance Sampling: Estimating Abundance of Biological Populations. Chapman \& Hall, New York.

Castro, R. y P. Soini. 1977. Field studies on Saguinus mystax and other callitrichids in Amazonian Peru. En: D. Kleiman (ed.): The Biology and Conservation of the Callitrichidae. Smithsonian Institution Press, Wahington, D.C., 73 - 78.

Castro, N.; F. Encarnación; L. Valverde; M. Ugamoto y E. Maruyama. 1990. Censo de primates no humanos en el suroriente peruano: Iberia e Iñapari (Departamento de Madre de Dios). En: N. castro (ed.): La Primatología en el Perú. Lima, Perú, 163 - 168.

Freese, C.; P. G. Heltne; R. Castro y G. Whitesides. 1982. Patterns and determinants of monkey densities in Peru and Bolivia, with notes on distribution. Int. J. Primatol. 3: 53 - 90.

Glander, K.; J. Tapia y A. Fachín. 1984. The impact of cropping on wild population of Saguinus mystax and Saguinus fuscicollis in Peru. Am. J. Primatol. 7: 89-97.

Goldizen, A. W. 1987. Tamarins and marmosets: communal care of offspring. En: B.B. Smuts, D. L. Cheney, R. M. Seyfarth, R. W. Wrangham y T. T. Struhsaker (ed.): Primates Societies. University of Chicago Press, Chicago, $34-43$.

Hershkovitz, P. 1977. Living New World monkeys (Plathyrrhini): With an introduction to primates. Vol. 1. University of Chicago Press. Chicago.

Heymann, E. W. 1990. Social behaviour and infant carrying in a group of moustached tamarins, Saguinus mystax (Primates: Platyrrhini: Callitrichidae), on Padre Isla, peruvian Amazonia. Primates 31(2): 183 - 196.

Heymann, E. W. 2000. Field observations of the golden- mantled tamarin, Saguinus tripartitus, on the Rio Curaray, peruvian Amazonia. Folia Primatologica 71:392-398.

Heymann, E. W.; F Encarnación y J. Canaquín. 2002. Primates of the Rio Curaray, northern peruvian Amazon. Int. J. Primatol. 23(1): 191 - 201.

Izawa, K.1978. Afield study of the ecology and behaviour of the black mantle-tamarin (Saguinus nigricollis). Primates 19: $241-274$.

Kostrub, C. E. 1997. Preliminary field observations of goldenmantled tamarins, Saguinus tripartitus, in eastern Ecuador. Neotropical Primates 5(4): 102-103.

Laake, J. F.; S. T. Buckland; D. R. Anderson y K. P. Burnham. 1994. Distances sampling: Abundance Estimation of Biological Populations - Distance User's Guide. Colorado Cooperative Fish \& Wildlife Research Unit, Colorado State University, Fort Collins, CO.

Puertas, P. F. Encarnación; R. Aquino y J. E. García. 1995. Análisis poblacional del pichico pecho anaranjado (Saguinus labiatus) en el Suroriente peruano. Neotropical Primates 3(1): 4-6.

Pook, A. G y G Pook. 1982. Polyspecific associations between Saguinus fuscicollis, Saguinus labiatus, and Callimico goeldii and other primates in nor-western Bolivia. Folia Primatologica 38: 196-216.

Rylands, A.; H. Schneider; A. Langguth; R. Mittermeier; C. Groves y E. Rodriguez-Luna. 2000. An assessment of the diversity of new world primates. Neotropical Primates 8(2): 61 - 93.

Soini, P. y M. Cóppula. 1995. Reporte Pacaya - Samiria: Investigaciones en la Estación Biológica Cahuana 1979 - 1994. Fundación para la Conservación de la Naturaleza, Iquitos, Perú.

Thorington, R. W. Jr. 1988. Taxonomic status of Saguinus tripartitus (Milne-Eduards, 1878). Am. J. Primatol. 15: 367 - 371.

Wilson, D. E.; F. R. Cole; J. D. Nichols; R. Rudran y M. Foster. 1996. Measuring and monitoring biological diversity: Standard methods for mammals. Smithsonian Institution Press, Washington. 